\title{
Quercetin Regulates Inflammatory Response of Retinal Ganglion Cells by Affecting the Tumor Necrosis Factor Receptor Associated Factor 6/Transforming Growth Factor- $\beta$-Activated Kinase-1 Signaling Pathway
}

\author{
ZIFENG DENG AND SHIBO XIONG ${ }^{1 *}$ \\ Department of Ophthalmology, Hunan Children's Hospital, Changsha 410007, Hunan Province, 'Wuhan Eyegood Ophthalmic \\ Hospital, Wuhan 430015, Hubei Province, China
}

\section{Deng et al.: Quercetin Protects Retinal Ganglion Cells}

\begin{abstract}
To investigate the protective effect of quercetin on the lipopolysaccharide-induced inflammatory response of retinal ganglion cell line 5 cells and to explore its possible molecular mechanism. Retinal ganglion cell line 5 cells were cultured in vitro and assigned into blank control group, lipopolysaccharide $(1.0 \mu \mathrm{g} / \mathrm{ml})$ induction group, lipopolysaccharide+low dose $(0.25 \mu \mathrm{g} / \mathrm{ml})$ quercetin group, lipopolysaccharide + medium dose $(0.5 \mu \mathrm{g} / \mathrm{ml})$ quercetin group and lipopolysaccharide+high dose $(1.0 \mu \mathrm{g} / \mathrm{ml})$ quercetin group. The relative survival rate of retinal ganglion cell line 5 cells was determined by cell counting kit-8 assay and the levels of inflammatory factors interleukin-6 and tumor necrosis factor-alpha were detected using enzymelinked immunosorbent assay kits. In addition, the levels of tumor necrosis factor receptor-associated factor 6, phosphorylation-transforming growth factor-beta-activated kinase 1 and transforming growth factorbeta-activated kinase 1 were measured by western blotting. The results of cell counting kit-8 assay revealed that quercetin could dramatically elevate the relative survival rate of retinal ganglion cell line 5 cells. The levels of inflammatory factors interleukin-6 and tumor necrosis factor-alpha in different quercetin groups were significantly lower than those in lipopolysaccharide induction group and the levels of interleukin-6 and tumor necrosis factor-alpha in cell supernatant declined with the increase of quercetin concentration in a concentration-dependent manner. In addition, the expression levels of tumor necrosis factor receptorassociated factor 6 and phosphorylation-transforming growth factor-beta-activated kinase 1 in different quercetin groups were remarkably lower than those in lipopolysaccharide induction group and they also declined with the increase of quercetin concentration in a concentration-dependent manner. Quercetin displays a protective effect against the lipopolysaccharide-induced inflammatory response of retinal ganglion cell line 5 cells and its mechanism is related to suppression of the activation of the tumor necrosis factor receptor-associated factor 6/transforming growth factor-beta-activated kinase 1 signaling pathway.
\end{abstract}

Key words: Quercetin, lipopolysaccharide, glaucoma, inflammatory response, retinal ganglion cell

Glaucoma is an eye disease characterized by intermittent or continuous increase in intraocular pressure ${ }^{[1]}$. Currently, there are nearly 600 million patients with glaucoma worldwide, of whom 8.4 million suffer from blindness $^{[2]}$. The pathogenesis of glaucoma, a kind of degenerative and blinding optic neuropathy caused by multiple factors, is complicated ${ }^{[3]}$ and its clinical manifestations are primarily optic atrophy, visual field damage and Retinal Ganglion Cell (RGC) apoptosis. Glaucoma ranks $2^{\text {nd }}$ among the eye diseases causing vision loss, seriously affecting the normal life and health of patients ${ }^{[4]}$. Optic nerve injury will further lead to endogenous inflammatory injury in RGCs, causing

*Address for correspondence

E-mail: 8113141@qq.com

November-December 2021 primary or secondary death of $\mathrm{RGCs}^{[5,6]}$. Quercetin mainly present in Chinese herbal medicines, fruits and vegetables is a natural flavonoid active substance, which possesses significant anti-inflammatory, antioxidation and anti-cancer effects and also plays a role similar to neurotrophic factors in neurons ${ }^{[7]}$. Quercetin has a protective effect against N-Methyl-D-Aspartic

This is an open access article distributed under the terms of the Creative Commons Attribution-NonCommercial-ShareAlike 3.0 License, which allows others to remix, tweak, and build upon the work non-commercially, as long as the author is credited and the new creations are licensed under the identical terms

Accepted 20 December 2021

Revised 06 July 2021

Received 07 April 2020

Indian J Pharm Sci 2021;83(6):1304-1308 
Acid (NMDA)-induced damage in BV-2 cells, whose mechanism may be related to its inhibition on the Tumor Necrosis Factor Receptor-Associated Factor 6 (TRAF6)/Transforming Growth Factor-Beta-Activated Kinase 1 (TAK1) signaling pathway. In this study, therefore, the inflammatory cell model was established using RGCs cultured in vitro to study the protective effect of quercetin against the Lipopolysaccharide (LPS) induced inflammatory response of RGC-5 cells and to preliminarily explore its possible mechanism. RGC-5 cells were purchased from Wuhan Biofavor Biotech Co., Ltd., Quercetin was purchased from Shaanxi Zhongxin Biotechnology Co., Ltd., Dulbecco's Modified Eagle Medium (DMEM) was purchased from Shanghai Guge Biological Co., Ltd., trypsin was purchased from Sigma (USA), Cell Counting Kit-8 (CCK-8) kits were purchased from MSK, Bicinchoninic Acid (BCA) protein concentration assay kits (batch No. P0012S-07) were purchased from Shanghai Guge Biological Co., Ltd., and Interleukin-6 (IL-6) and Tumor Necrosis Factor-Alpha (TNF- $\alpha$ ) assay kits were purchased from Shanghai Huamei Biotechnology Co., Ltd. Antibody diluent (batch No. C154855) was bought from Shanghai Jinuo Biotechnology Co., Ltd., Electrogenerated Chemiluminescence (ECL) solution was bought from Sigma, Beta $(\beta)$-actin, TRAF6, TAK1 and Phosphorylation-Transforming Growth Factor- $\beta$ Activated Kinase 1 (p-TAK1) antibodies were all bought from Cell Signaling Technology (USA), goat anti-rabbit Immunoglobulin $\mathrm{G}$ (IgG) secondary antibodies were bought from LI-COR Biosciences (USA), a single-person clean bench and a $\mathrm{CB} 15 \mathrm{C} 02$ cell incubator were bought from Beijing Liuyi Instrument Factory, a Victor3 1420 Multilable Counter microplate reader was bought from BD (USA), an HD3000 gel imager was bought from Shanghai Shangtian Precision Instrument Co., Ltd., and a $7230 \mathrm{G}$ ultraviolet visible spectrophotometer was bought from Shanghai Jinghua Technology Instrument Co., Ltd. RGC-5 cells were cultured in complete DMEM in a $5 \% \mathrm{CO}_{2}$ constant humidity incubator at $37^{\circ}$. When about $90 \%$ of the cells adhered to the wall, the old medium was discarded and the cells were washed twice with sterile PBS and digested with trypsin. After the cells became round, the digestion was terminated with medium, followed by centrifugation and resuspension. Finally, the cells were sub-cultured at 1:3 ratio. RGC-5 cells in the logarithmic growth were harvested, digested, centrifuged and resuspended and the cell density was adjusted. Then the cells were inoculated into a 96 -well plate $\left(1 \times 10^{4}\right.$ cells/ well) and cultured for $24 \mathrm{~h}$. After the medium was discarded, quercetin at different concentrations $(0.25$, 0.5 and $1.0 \mathrm{mg} / \mathrm{ml})$ and LPS $(1.0 \mu \mathrm{g} / \mathrm{ml})$ were added for intervention for $24 \mathrm{~h}$. Then the medium in the plate was aspirated and serum-free medium containing $10 \mu \mathrm{l}$ of CCK-8 reagent was added into each well for culture for $1 \mathrm{~h}$. Finally, the optical density of each well was measured at a wavelength of $450 \mathrm{~nm}$ using a microplate reader. RGC-5 cells in the logarithmic growth were harvested, washed, digested and resuspended. They were inoculated into a 6 -well plate $\left(5 \times 10^{5}\right.$ cells/well $)$, with 6 replicates in each group, cultured in the $5 \% \mathrm{CO}_{2}$ incubator at $37^{\circ}$ overnight and then subjected to intervention with quercetin at different concentrations $(0.25,0.5$ and $1.0 \mathrm{mg} / \mathrm{ml})$ and LPS $(1.0 \mu \mathrm{g} / \mathrm{ml})$ for $24 \mathrm{~h}$. The cell supernatant was harvested to detect the levels of inflammatory factors IL- 6 and TNF- $\alpha$ using ELISA kits in strict accordance with the instructions. The cells were lysed and the protein concentration was measured. The lysate was added with loading buffer and heated at $1000^{\circ}$ for $10 \mathrm{~min}$. Then $40 \mu \mathrm{g}$ of proteins were loaded in each well and separated by $12 \%$ Sodium Dodecyl Sulphate-Polyacrylamide Gel Electrophoresis (SDSPAGE) (voltage: $70 \mathrm{~V}$ for spacer gel and $120 \mathrm{~V}$ for separation gel). After separation, the protein was transferred onto the nitrocellulose membrane at 275 $\mathrm{mA}$ for $60 \mathrm{~min}$ and the membrane was blocked with $5 \%$ skim milk powder at room temperature for $1 \mathrm{~h}$. The primary antibodies were used for incubation overnight and the secondary antibodies were used for incubation at room temperature for $1 \mathrm{~h}$. Finally, the membrane was washed for 3 times followed by color development and imaging. Statistical Package for the Social Sciences (SPSS) 26.0 software was used for statistical analysis. Normally distributed measurement data were expressed as mean \pm standard deviation and compared between two groups using Least Significant Difference (LSD)-t test in analysis of variance, $\mathrm{p}<0.05$ was considered statistically significant. The cell survival rate in LPS group was significantly lower than that in control group $(\mathrm{p}<0.05)$, indicating that LPS can inhibit the proliferation of RGC-5 cells. After intervention with low, medium and high dose quercetin, the cell proliferation was obviously enhanced and the relative survival rate also rose with the increase in the drug concentration, showing concentration dependence (fig. 1). The levels of IL- 6 and TNF- $\alpha$ in cell supernatant in LPS group were significantly higher than those in control group $(p<0.05)$, indicating that LPS can induce the release of IL- 6 and TNF- $\alpha$ in RGC-5 cells. After intervention with low, medium and high dose quercetin, the levels of inflammatory factors in RGC-5 cells significantly 
declined and they were also decreased with the increase in the drug concentration, showing concentration dependence (fig. 2). The levels of TRAF6 and p-TAK1 in LPS group were higher than those in control group $(p<0.05)$. After intervention with low, medium and high dose quercetin, the levels of TRAF6 and p-TAK1 in RGC-5 cells declined with statistically significant differences and they were also decreased with the increase in the drug concentration, showing concentration dependence (fig. 3). The retina is an important photoreception organ able to perceive visual signals followed by further processing. In the case of damage, however, visual disorders or blindness will be

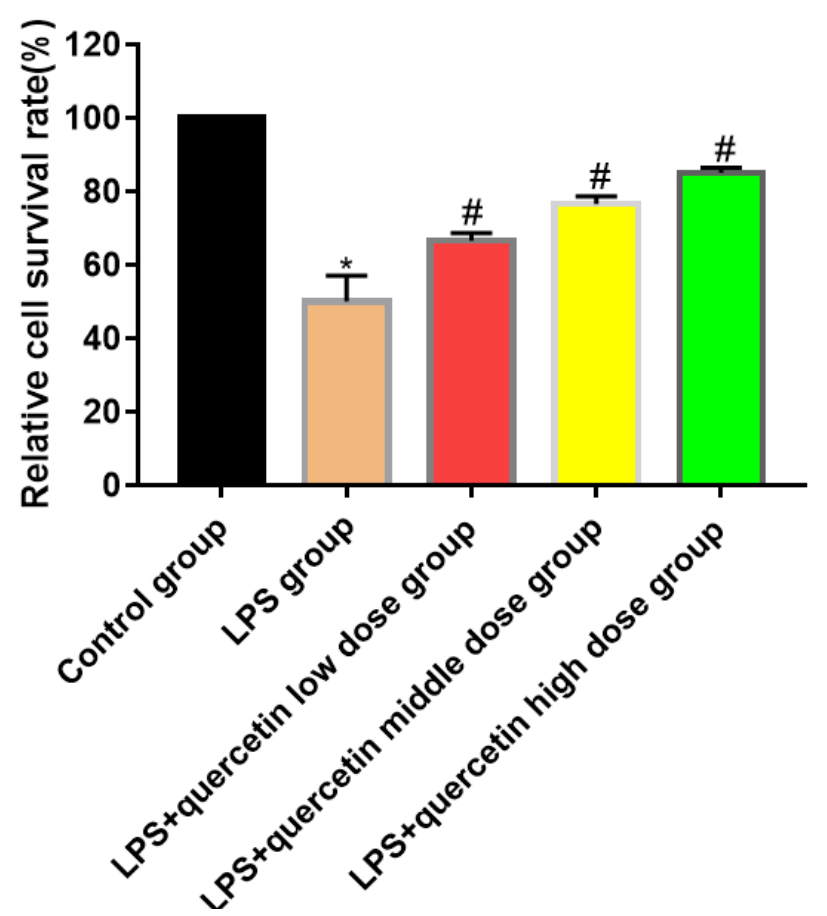

Fig. 1: Effects of quercetin on the survival rate of RGC-5 cells Note: * $p<0.05$ vs. control group; ${ }^{\#} \mathbf{p}<0.05$ vs. LPS group

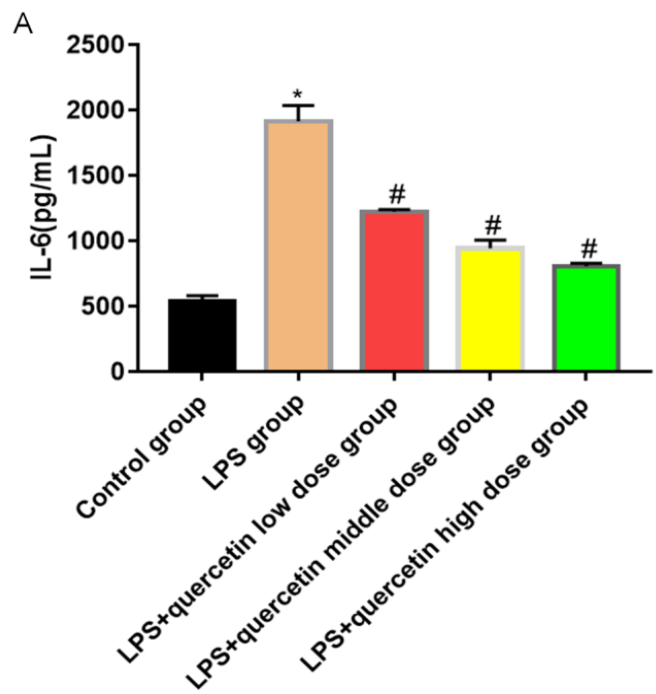

caused $^{[8]}$. RGCs are cells responsible for retinal signal output, which process and transmit the signals through the retinal neural circuit to the visual center in the form of action potentials, further producing normal vision ${ }^{[9,10]}$. Under a variety of stimuli, retinal oxidative stress will be caused, the Nuclear Factor Kappa B (NF-kB) signaling pathway will be activated, the transcription of downstream target genes will be initiated and the interaction between inflammatory factors (IL-6 and TNF- $\alpha$ ) and adhesion molecules will be induced, further leading to pathological processes such as retinal capillary occlusion and non-perfusion, endothelial injury and neovascularization ${ }^{[11,12]}$. Therefore, the eye can be well protected from damage by inhibiting the inflammatory response of RGCs. Traditional Chinese medicine has definite efficacy in the treatment of glaucoma. Quercetin is a natural flavonoid active substance and flavonoids can ameliorate the inflammatory factor-induced inflammatory injury of neurons, so quercetin has a good pharmacological effect in the prevention and treatment of inflammatory diseases $^{[13]}$. In the present study, the effects of quercetin at different concentrations on RGC-5 cells were observed by CCK-8 assay. It was found that $0.1-2.0$ $\mathrm{mg} / \mathrm{ml}$ quercetin had no significant effects on the proliferative activity and morphology of RGC-5 cells. Therefore, RGC-5 cells were treated with $0.25,0.5$ and $1.0 \mathrm{mg} / \mathrm{ml}$ quercetin in this study. The inflammatory cell model was established by LPS in vitro and the protective effect of quercetin against inflammatory injury in RGC-5 cells was observed. The results revealed that quercetin could greatly increase the relative survival rate of $\mathrm{RGC}-5$ cells and the inflammatory factors IL- 6 and TNF- $\alpha$ could be

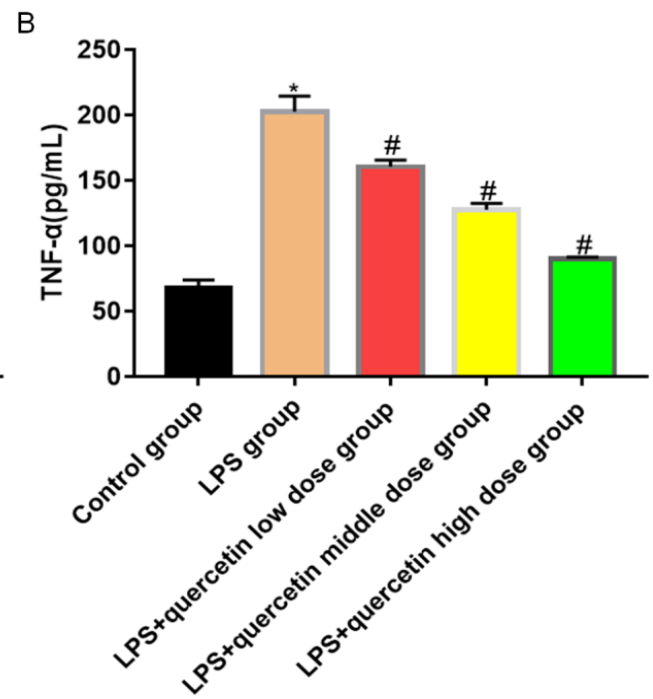

Fig. 2: Effects of quercetin on levels of (A) IL-6 and (B) TNF- $\alpha$ in supernatant of RGC-5 cells Note: *p<0.05 vs. control group; ${ }^{*} \mathbf{p}<\mathbf{0 . 0 5}$ vs. LPS group 
A

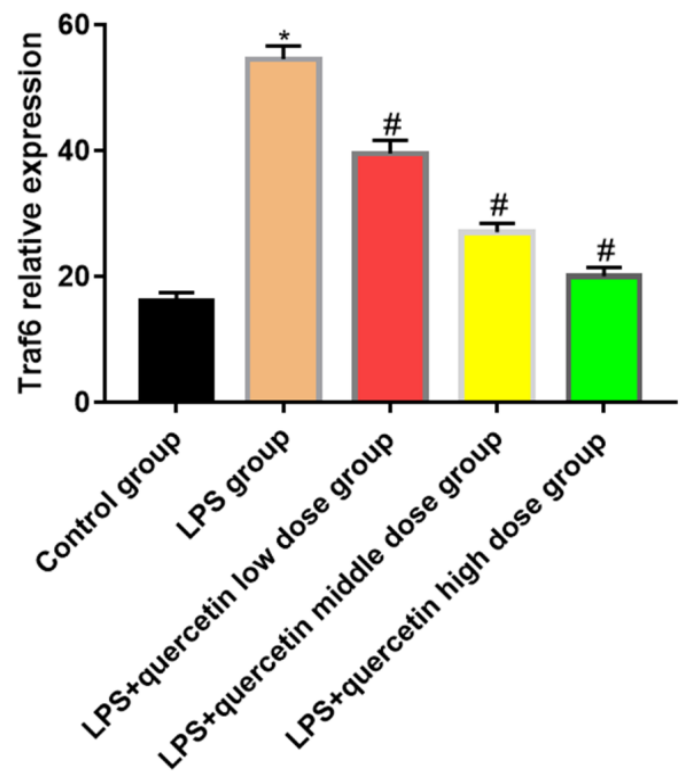

B

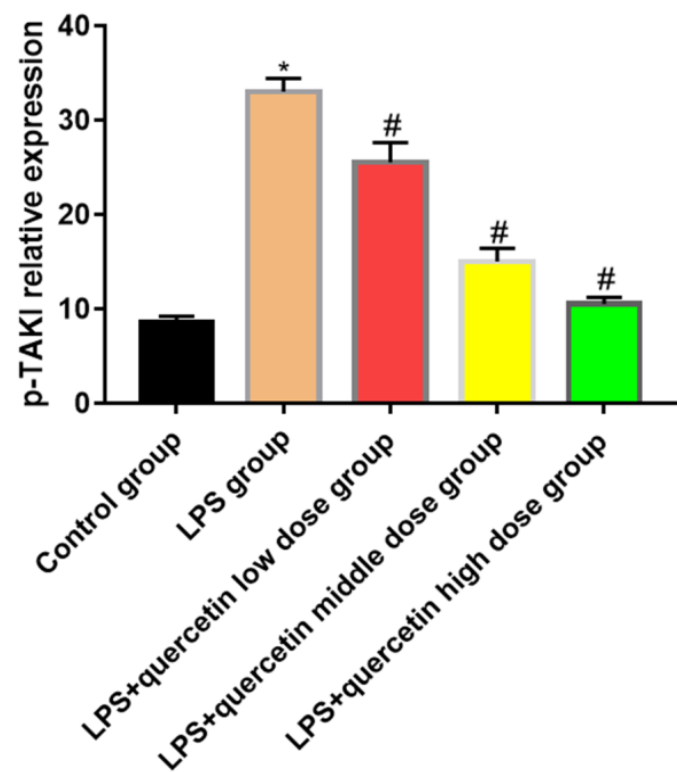

Fig. 3: Effects of quercetin on the TRAF6/TAK1 signaling pathway of RGC-5 cells Note: *p<0.05 vs. control group; ${ }^{*}<<0.05$ vs. LPS group

obviously reduced by different doses of quercetin in a concentration dependent manner. TRAF6, an adapter protein, can transmit signals mediated by a variety of receptors on the membrane, including those mediated by the toll-interleukin-1 receptor family ${ }^{[14]}$. LPS can cause the ubiquitination of TRAF6 and then TRAF6 together with TAK1 forms complexes to further activate IkappaB kinase kinases (IKKs) and promote the transfer of NF- $\mathrm{NB}$ to the nucleus, ultimately resulting in inflammatory response, including the increase in the levels of IL- 6 and TNF- $\alpha$. It can be seen that inhibiting the TRAF6/TAK1 signaling pathway can reduce the production of inflammatory factors and alleviate the inflammatory injury of cells. Besides, the expression of TRAF6 shows an increasing trend in injury of RGCs, which may be related to the pathogenesis of glaucoma ${ }^{[15]}$. In the present study, it was found that quercetin could significantly inhibit the expressions of TRAF6 and p-TAK1 and weaken the activation of the TRAF6/ TAK1 signaling pathway, further reducing the inflammatory response. In conclusion, quercetin can exert an anti-inflammatory effect through inhibiting the activation of the TRAF6/TAK1 signaling pathway and reducing the inflammatory factors IL- 6 and TNF- $\alpha$.

\section{Conflict of interests:}

The authors declared no conflicts of interest.

\section{REFERENCES}

1. Qiao C, Jia H, Zhang H, Wang H, Liang J, Song J, et al. Coding variants in HOOK2 and GTPBP3 may contribute to risk of primary angle closure glaucoma. DNA Cell Biol
2020;39(6):949-57.

2. Kyei S, Obeng PA, Kwarteng MA, Assiamah F. Epidemiology and clinical presentation of glaucoma in a referral facility in Ghana: Any lessons for public health intervention? Plos one 2021;16(1):e0245486.

3. Tao Y, Tham YC, Chee ML, Majithia S, Thakur S, Soh ZD, et al. Profile of retinal nerve fibre layer symmetry in a multiethnic Asian population: the Singapore Epidemiology of Eye Diseases study. Br J Ophthalmol 2020;104(6):836-41.

4. Lee JW, Chan PP, Zhang X, Chen LJ, Jonas JB. Latest developments in normal-pressure glaucoma: diagnosis, epidemiology, genetics, etiology, causes and mechanisms to management. Asia Pac J Ophthalmol 2019;8(6):457-68.

5. Fernández-Albarral JA, Salazar JJ, de Hoz R, Marco EM, Martín-Sánchez B, Flores-Salguero E, et al. Retinal Molecular Changes Are Associated with Neuroinflammation and Loss of RGCs in an Experimental Model of Glaucoma. Int J Mol Sci 2021;22(4):2066.

6. Huang TL, Wen YT, Ho YC, Wang JK, Lin KH, Tsai RK. Algae oil treatment protects retinal ganglion cells (RGCs) via ERK signaling pathway in experimental optic nerve ischemia. Mar Drugs 2020;18(2):83.

7. Albatany M, Meakin S, Bartha R. The Monocarboxylate transporter inhibitor Quercetin induces intracellular acidification in a mouse model of Glioblastoma Multiforme: In vivo detection using magnetic resonance imaging. Invest New Drugs 2019;37(4):595-601.

8. Yamamoto N, Hiramatsu N, Ohkuma M, Hatsusaka N, Takeda $\mathrm{S}$, Nagai $\mathrm{N}$, et al. Novel Technique for Retinal Nerve Cell Regeneration with Electrophysiological Functions Using Human Iris-Derived iPS Cells. Cells 2021;10(4):743.

9. Zhang Y, Zhang S, Xia Y, Ji Y, Jiang W, Li M, et al. In vivo evaluation of retinal ganglion cells and optic nerve's integrity in large animals by multi-modality analysis. Exp Eye Res 2020;197:108117.

10. Kumar V, Ali Shariati M, Mesentier-Louro L, Jinsook Oh A, Russano K, Goldberg JL, et al. Dual Specific Phosphatase 14 Deletion Rescues Retinal Ganglion Cells and Optic Nerve Axons after Experimental Anterior Ischemic Optic Neuropathy. 
Curr Eye Res 2021;46(5):710-8.

11. Zhang HJ, Xing YQ, Jin W, Li D, Wu K, Lu Y. Effects of curcumin on interleukin-23 and interleukin-17 expression in rat retina after retinal ischemia-reperfusion injury. Int J Clin Exp Pathol 2015;8(8):9223-31.

12. Mohamed IN, Soliman SA, Alhusban A, Matragoon S, Pillai BA, Elmarkaby AA, et al. Diabetes exacerbates retinal oxidative stress, inflammation and microvascular degeneration in spontaneously hypertensive rats. Mol Vis 2012;18:1457-66.

13. Zhou YS, Xu J, Peng J, Liu Y, Qin GY, Yang YJ, et al. Effect of Qingguang'an II on expressions of OX42 protein and IL$1 \beta$ mRNA of retinal microglia cells of rats with chronic high intraocular pressure. Int J Ophthalmol 2018;11(2):326-8.

14. Greene JA, Portillo JA, Lopez Corcino Y, Subauste CS. CD40TRAF signaling upregulates CX3CL1 and TNF- $\alpha$ in human aortic endothelial cells but not in retinal endothelial cells. PloS one 2015;10(12):e0144133.

15. Wang R, Sun Q, Xia F, Chen Z, Wu J, Zhang Y, et al. Methane rescues retinal ganglion cells and limits retinal mitochondrial dysfunction following optic nerve crush. Exp Eye Res 2017;159:49-57. 